\title{
Pharmakologische Beeinflussungsmöglichkeiten der Tremorin- und Arecolin-Hypothermie
}

\author{
Alfred H. Staib \\ Pharmakologisches Institut der Medizinischen Akademie \\ „Carl Gustav Carus", Dresden
}

\begin{abstract}
Possible pharmacological influences of tremorine- and arecoline-hypothermia. Both tremorine and arecoline cause a drop in body temperature in small laboratory animals (mice, rats, guinea pigs) as well as tremor, rigor and peripheric effects. The purpose of this paper is (1) to characterize the up-until-now unknown hypothermic effect of arecoline, as compared with tremorine, and (2) to check the action of drugs used in Parkinson's disease and others on this hypothermia in mice in order to obtain information on the action of tremorine and arecoline on thermoregulation and on their antagonists. The relations between dose and body temperature of male mice kept at different ambient temperatures after intraperitoneal injection of arecoline or tremorine and the influences of antagonists (in view of tremor) on hypothermia have been studied. The hypothermic action following the use of tremorine is more continuous and more pronounced than that following arecoline. Judged by the same degree of body temperature depression, the effect of tremorine is five to 10 times stronger than that of arecoline. Scopolamine, atropine, benactycine and trihexyphenidyl block the hypothermic effect of tremorine and arecoline; hypothermia after arecoline is nor influenced by caramiphen; antiparkin, butylscopolamine, as well as the phenothiazinederivatives ethopropazine, promethazine and diethazine, are also ineffective; methylatropine reduces the arecoline-hypothermia only in high doses. Hypothermia after arecoline and tremorine is mainly based on a central action. The effects of several central cholinolytics and the antihypothermic inefficiency of phenothiazines, used in Parkinson's disease and against motoric effects of tremorine and arecoline, demonstrate a difference within this group of drugs. The trigger in hypothermic action is a cholinergic one.
\end{abstract}

\section{EINLEITUNG}

Tremorin [1,4-Dipyrolidino-butin-(2)] ruft neben Veränderungen der Motorik (Tremor) und peripher muskarinartigen Wirkungen eine ausgeprägte Hypothermie hervor. Bereits in der ersten Veröffentlichung von Everett, Blockus \& SHEPPERd (1956) wird auf diesen Effekt der in der Folgezeit beim Screening von Antiparkinsonstoffen bewährten Substanz hingewiesen. Diese Hypothermie ist mehrfach mit dem Ziel der Aufklärung des Mechanismus untersucht (Everetr 1964, Farquharson \& Johnston 1959, Ferrari \& Gessa 1964, Keranen, Zaratzian \& Coleman 1961) und zur Klärung allgemein-thermoregulatorischer Probleme (MAIKel, STERn \& Brodie 1964) herangezogen worden. Das seit langem in der experimentellen Pharmakologie bekannte 
Arecolin (das Hauptalkaloid der Betelnuß - Areca) gewinnt in letzter Zeit im Zusammenhang mit seinen zentralen. Wirkungen erneut an Interesse (HEALY \& JENNEY 1959, Herz 1962, Oelszner unpubliziert, Votava 1962). Die nach Tremorin auftretenden und einzelnen Symptomen des Parkinsonsyndroms ähnelnden Erscheinungen am Tier können auch durch Arecolin hervorgerufen und in beiden Fällen durch Antiparkinsonmittel gehemmt werden. Auch nach Arecolin wird eine Senkung der Körpertemperatur bei Mäusen beobachtet (ADLER 1964). Damit ist qualitativ eine weitgehende Parallelität der Wirkung $\mathrm{z}$ wischen Tremorin und Arecolin gegeben.

Mit den vorliegenden Untersuchungen sollen die Entstehungsbedingungen beider Hypothermieformen sowie ihre pharmakologischen Kennzeichen erfaßt werden. Es wird von der Beeinflußbarkeit der oben genannten motorischen Effekte durch zentrale Cholinolytika und einiger anderer Stoffe, die unter dem Begriff Antiparkinsonmittel zusammengefaßt werden, ausgegangen. Ziel unserer Versuche war, den Grad der Vergleichbarkeit der Temperatureffekte beider Substanzen zu erfassen und gegebenenfalls Rückschlïsse auf den Entstehungsmechanismus zu ziehen.

\section{METHODE}

Für die Versuche wurden männliche Albino-Mäuse im Gewicht von 16 bis $24 \mathrm{~g}$, die unter Standardbedingungen gehalten wurden, verwendet. Die Untersuchungen gliedern sich in zwei Gruppen: Die erste diente dem Vergleich der hypothermen Wirksamkeit von Tremorin und Arecolin und fand mit der Ermittlung von Dosis-Wirkungs-Kurven ihren Abschluß. In der zweiten Gruppe wurde die Wirksamkeit hypothermisierender Dosen beider Stoffe unter dem Einfluß von Antiparkinsonmitteln untersucht.

Untersuchungsgruppe I. Die Mäuse wurden 20 min vor Versuchsbeginn in einem thermostatisch geregelten Raum (Kühlzelle) gebracht, der auf $10^{\circ}, 15^{\circ}, 20^{\circ}, 25^{\circ}$ und $30^{\circ} \mathrm{C}$ eingestellt war. Die Rektaltemperaturen der in Einzelkäfigen befindlichen Tiere wurden mit Thermistoren gemessen. Die Widerstandsänderungen der für die Messung im Rectum präparierten Thermistoren wurden mittels einer Brückenschaltung erfaßt und über ein Mikro-Amperemeter registriert. Nach Eichung waren die absoluten Temperaturen mit einer Genauigkeit von $\pm 0,03^{\circ} \mathrm{C}$ ablesbar. Nach Eingewöhnung der Tiere und Ermittlung der Temperaturleerwerte erfolgte die intraperitoneale Injektion geometrisch abgestufter Dosen des in 0,9\% iger NaCl-Lösung gelösten Arecolinhydrobromids beziehungsweise Tremorins (verwendet wurden nur Tiere mit einer Rektaltemperatur $>36^{\circ} \mathrm{C}$ nach Eingewöhnung). Nach der Injektion wurden die Rektaltemperaturen bei den Tremorintieren in 10-Minuten-Abständen und bei den Arecolintieren über 30 min in 5-Minuten-Abständen, danach ebenfalls in 10-Minuten-Abständen insgesamt 120 min bestimmt. Zur Ermittlung wirksamer Dosen nach einem Alles-oderNichts-Verfahren werteten wir die Tiere als hypotherm, deren Rektaltemperatur $\leqq 33,0^{\circ} \mathrm{C}$ betrug. Diese Tiere mit positiver Reaktion wurden für jeden Meßzeitpunkt und für jede Dosis prozentual ermittelt. Im Falle eines erkennbaren dosisabhängigen Reaktionsverlaufs wurde nach dem Verfahren von Lichtrield \& Wilcoxon (1949) die Dosis bestimmt, bei der $84 \%$ der Tiere das gewählte Kriterium erfüllten ( $\leqq 33,0^{\circ} \mathrm{C}$ ).

Untersuchungsgruppe II. Die $E_{84}$ bei einer Umgebungstemperatur von $10^{\circ} \mathrm{C}$ wurde dem zweiten Teil der Untersuchungen zugrunde gelegt. Diese Dosis erhiel- 
ten die in gleicher Weise vorbereiteten Tiere intraperitoneal. Die ED 84 Tremorin war auf eine Temperaturbestimmung $75 \mathrm{~min}$ nach der Injektion berechnet, 50 min nach der Applikation erhielten die Tiere subcutan eine Dosenfolge der zu prüfenden Antiparkinsonstoffe, 25 min später wurde die Rektaltemperatur zweimal gemessen. Die mit einer $\mathrm{ED}_{84}$ Arecolin (berechnet auf die 15. Minute nach der Injektion) behandelten Tiere erhielten die zu prüfenden Stoffe subcutan $15 \mathrm{~min}$ vor Arecolin, und die Rektaltemperatur wurde 15 min nach Arecolin gemessen. Jeder Versuchsgruppe liefen Kontrollen parallel, die anstelle der Antiparkinsonstoffe 0,9\% oige $\mathrm{NaCl}$-Lösung subcutan erhielten. Es wurde durch Bestimmung der Temperaturmittelwerte für jede Stoffgruppe für die effektiven Dosen die Beeinflussung des Hypothermieeffektes im Vergleich zu den Kontrollversuchen mittels $t$-Test geprïf. Die Tiere, deren Rektaltemperaturen nicht auf 33,0 $0^{\circ} \mathrm{C}$ oder darunter sanken, wurden als positiv im Sinne eines Antagonismus gegenüber der hypothermen Wirkung betrachtet. Graphisch wurde nach LichTFIELD \& WILCoxon die Dosis ermittelt, bei der nur noch $16 \%$ der Tiere den vollen Hypothermieeffekt aufwiesen.

\section{ERGEBNISSE}

Arecolin bewirkt ebenso wie Tremorin bei der Maus eine dosisabhängige Senkung der Körpertemperatur, die bei gleicher Dosis mit zunehmender Umgebungstemperatur geringer wird (Abb, 1). Der Effekt von Arecolin hält kürzere Zeit an, was besonders bei den höheren Umgebungstemperaturen zum Ausdruck kommt. Außerdem liegen die für den gleichen Effekt nötigen Dosen bei Arecolin bedeutend höher. Tabelle 1 gibt die Effektivdosen Tremorin und Arecolin bei verschiedenen Umgebungstemperaturen an.

Mit den ermittelten Dosen können selbst bei den $\mathrm{ED}_{15}$-Werten (die in Tab. 1 nicht angeführt sind) ein deutlicher Tremor sowie peripher muskarinartige Wirkungen ausgelöst werden. Bei $+30^{\circ}$ und $25^{\circ} \mathrm{C}$ Raumtemperatur konnte für Arecolin infolge der geringen temperatursenkenden Wirkung keine Dosis-Wirkungs-Beziehung ermittelt werden, $\mathrm{da}$ in diesem Bereich bereits toxische Effekte auftraten; nach Tremorin liegt bei $25^{\circ} \mathrm{C}$ die $\mathrm{ED}_{84} 90$ min nach der Injektion mit $7,60 \mathrm{mg} / \mathrm{kg}$ deutlich über den Werten bei den tieferen Umgebungstemperaturen.

Die Beeinflußbarkeit der Hypothermie durch Antiparkinsonstoffe ist bei beiden Substanzen gleichsinnig (Tab. 2). Zentrale Cholinolytika wie Atropin, Scopolamin und

\section{Tabelle 1}

Dosen von Arecolin und Tremorin in $\mathrm{mg} / \mathrm{kg}$ i. p., die bei $84 \%$ der Mäuse zu Rektaltemperaturen von oder unter $33,0^{\circ} \mathrm{C}$ führen. Abhängigkeit von der Zeit nach der Injektion und der Umgebungstemperatur

\begin{tabular}{|ccccccc|}
\hline $\begin{array}{c}\text { Raum- } \\
\text { temp. } \\
\left(^{\circ} \mathrm{C}\right)\end{array}$ & $30^{\prime}$ & $\begin{array}{c}\text { ED } 84 \text { Tremorin } \\
60^{\prime}\end{array}$ & $90^{\prime}$ & $15^{\prime}$ & $25^{\prime}$ & $60^{\prime}$ \\
\hline 10 & 3,50 & 3,90 & 3,60 & 28,0 & 28,0 & $\%$ \\
15 & 5,40 & 3,35 & 3,40 & 38,0 & 58,0 & 52,0 \\
20 & 5,30 & 3,85 & 3,65 & 44,0 & 37,0 & $\%$ \\
25 & $\%$ & $\%$ & 7,60 & $\%$ & $\%$ & $\%$ \\
\hline
\end{tabular}


Benactyzin hemmen die Senkung beziehungsweise führen zur Normalisierung der gesenkten Körpertemperatur, in hohen Dosen zeigen diesen Effekt auch Trihexyphenidyl und in der Tremorinreihe Caramiphen. Alle anderen untersuchten Verbindungen verhindern die Hypothermie nicht, einige (Diaethazin) führen sogar zu einer Verstärkung
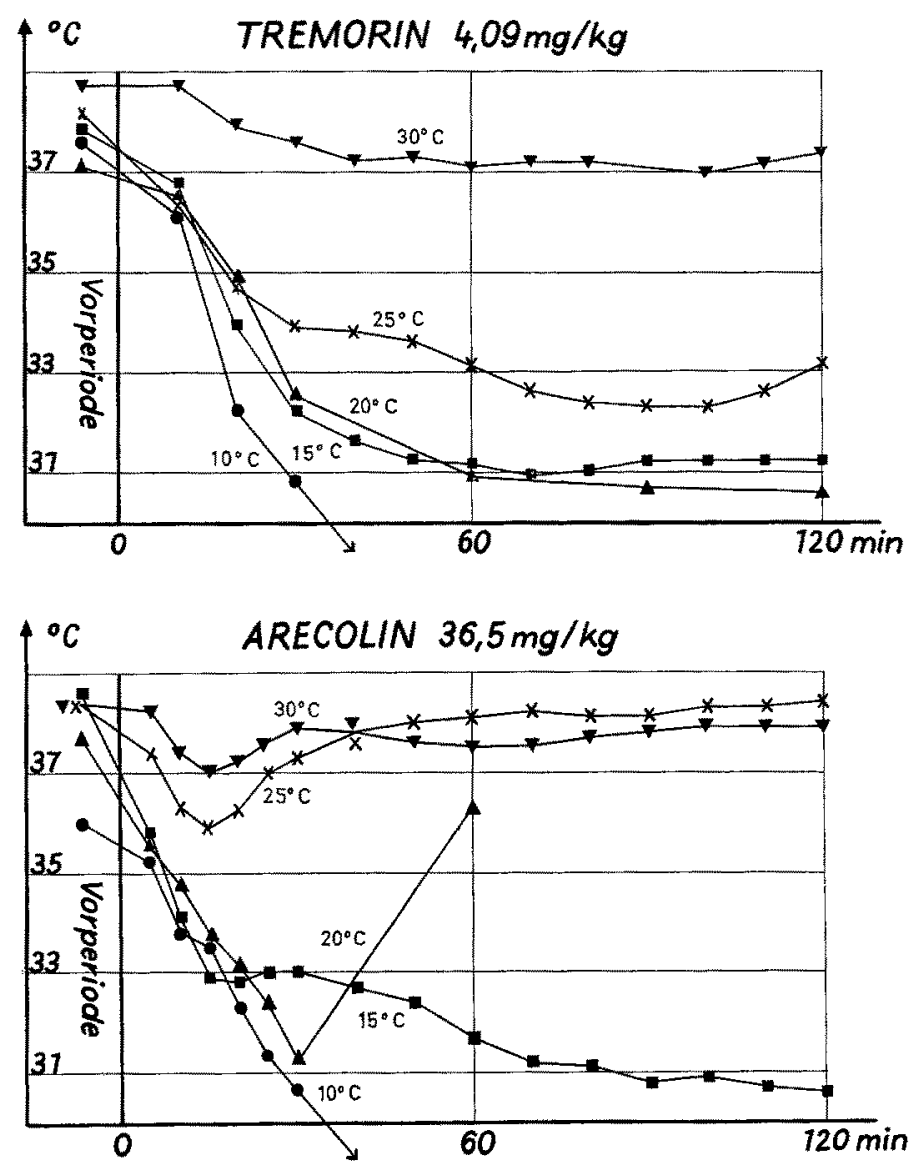

Abb. 1: Hypothermieeffekt einer vergleichbaren Tremorin- und Arecolindosis bei der Maus. Umgebungstemperaturen zwischen $10^{\circ}$ und $30^{\circ} \mathrm{C}$. Körpertemperaturen: Mittelwerte

des Effekts. Die quaternierten. Derivate des Atropins und Scopolamins hemmen nur in sehr hohen Dosen die Senkung der Körpertemperatur. Ihre Wirksamkeit ist jedoch variabel, so daß besonders bei Methylatropin in einigen Versuchsreihen auch bei mittleren Dosen eine deutliche Wirkung nachweisbar war. Die Effektivdosen der Stoffe, bei denen eine klare Dosis-Wirkungs-Beziehung zu ermitteln war, werden in Tabelle 3 dargestellt. 
Tabelle 2

Temperaturmittelwerte nach Gabe hypotherm wirksamer Tremorin- und Arecolindosen (ED 84 i. p., Umgebungstemperatur $10^{\circ} \mathrm{C}$ ) in Kombination mit Antiparkinsonmitteln (Dosis in $\mathrm{mg} / \mathrm{kg}$ s. c. für Tremorinreihe/Arecolinreihe - T./A.); $\mathrm{t}$-Test zu den Kontrollen $++\mathrm{p}$ $<0,01,{ }^{+} \mathrm{p}<0,05$

\begin{tabular}{|lccc|}
\hline Pharmaka & $\begin{array}{c}\text { Dosis } \\
\text { T.A. }\end{array}$ & $\begin{array}{c}\text { Tremorin } \\
\text { Rectal-Temp. } \\
75^{\prime} \mathrm{p} . \mathrm{i} .\end{array}$ & $\begin{array}{c}\text { Arecolin } \\
\text { Rectal-Temp. } \\
15^{\prime} \mathrm{p} . \mathrm{i} .\end{array}$ \\
\hline Scopolamin & $1,3 / 1,3$ & $35,5 \pm 0,22++$ & $36,9 \pm 0,05++$ \\
Atropin & $5,0 / 5,0$ & $34,3 \pm 0,30++$ & $35,8 \pm 0,20++$ \\
Butylscopolamin & $14,6 / 14,6$ & $32,6 \pm 0,43$ & $32,9 \pm 0,43$ \\
Methylatropin & $12,5 / 12,5$ & $<30,0$ & $34,4 \pm 0,42++$ \\
Benactyzin & $0,69 / 1,02$ & $34,6 \pm 0,40++$ & $34,3 \pm 0,05++$ \\
Trihexyphenidyl & $54,4 / 15,2$ & $33,3 \pm 0,43++$ & $35,2 \pm 0,28++$ \\
Caramiphen & $10,0 / 10,0$ & $33,5 \pm 0,61+$ & $33,5 \pm 0,38$ \\
Antiparkin & $10,0 / 10,0$ & $32,8 \pm 0,53$ & $34,1 \pm 0,43$ \\
Diaethazin & $20,0 / 20,0$ & $30,2 \pm 0,07(++)$ & $33,7 \pm 0,32$ \\
Promethazin & $20,0 / 19,5$ & 20,30 & $31,5 \pm 0,53$ \\
Aethopropazin & $20,0 / 20,0$ & $31,9 \pm 0,28$ & $33,6 \pm 0,40$ \\
Kontrollen & $0,1 \mathrm{ml} /$ & $32, \pm \pm 0,26$ & $32,6 \pm 0,23$ \\
(0,9\%0 NaCl) & $10 \mathrm{~g} \mathrm{Maus}$ & $(\mathrm{n}=59)$ & $(\mathrm{n}=59)$ \\
\hline
\end{tabular}

Tabelle 3

Dosen von Antiparkinsonmitteln (mg/kg s. c.), die eine Hypothermie-ED84 Arecolin beziehungsweise Tremorin zu einer $E D_{16}$ reduzieren

\begin{tabular}{|lcc|}
\hline Pharmaka & $\begin{array}{c}\text { Tremorin } \\
\mathrm{ED}_{84} \rightarrow \mathrm{ED}_{16}\end{array}$ & $\begin{array}{c}\text { Arecolin } \\
\mathrm{ED}_{84} \rightarrow \mathrm{ED}_{16}\end{array}$ \\
\hline Atropin & 4,30 & 2,20 \\
Scopolamin & 1,34 & 0,37 \\
Benactycin & 0,60 & 0,36 \\
Trihexyphenidyl & 90,0 & 10,4 \\
\hline
\end{tabular}

\section{DISKUSSION}

Die bisherigen Untersucher der Tremorinhypothermie weisen auf deren zentrale Genese und die Beteiligung eines cholinergen Mechanismus hin. So waren nach EvErETt, Blockus \& Shepperd (1956) sowie von Keranen, Zaratzian \& Coleman 1961) Atropin, Scopolamin und Benactyzin protektiv gegenüber der Hypothermie wirksam. Tremorin erhöht den Acetylcholingehalt des Gehirns signifikant, ein Effekt, der durch Atropin gehemmt werden kann (PEPEU 1963). Farquharson \& Johnston (1959) erwähnen Caramiphen als wirksam gegen die Tremorinhypothermie der Maus. Schließlich wiesen Ferrari \& Gessa (1964) nach, daß die Senkung der Körpertemperatur nach Tremorin bei der Ratte durch den Benzhydrylabkömmling Orphenadrin nur unbedeutend und durch die Phenothiazinderivate Profenamin und Diaethazin überhaupt nicht beeinflußbar ist. Die zentrale Auslösung der Störung der Wärmeregulation wird unter anderem daraus abgeleitet, daß die quaternierten Derivate des Atropins wirkungslos sind. Von anderen Autoren konnte jedoch an der Ratte durch Methylatropin eine, wenn 
auch gegenüber Atropin geringere Wirksamkeit auf die durch Tremorin ausgelöste Körpertemperatursenkung ermittelt werden (MAIKEL, STERN \& Brodie 1964).

Untersuchungen über die Arecolinhypothermie liegen unseres Wissens bisher noch nicht vor. Zentrale Effekte dieses Stoffes können bereits nach relativ niedrigen Dosen nachgewiesen werden. So ermittelten Herz (1962) 1 bis $4 \mathrm{mg} / \mathrm{kg}$, OenszNer (unpubliziert) $5,2 \mathrm{mg} / \mathrm{kg}$ als Effektivdosen für die Arecolinanalgesie; $2,0 \mathrm{mg} / \mathrm{kg}$ subcutan führen zur Hemmung bedingter Reflexe (HEaly \& Jenney 1959). Tremor tritt erst nach höheren Dosen auf. Die ED $\mathrm{ED}_{84}$ (Tremor) bei der Maus beträgt zum Beispiel 22,5 mg/kg (i.p.). Die zentralen Wirkungen sind jedoch durch die gleichen Stoffe hemmbar, die auch gegenüber Tremorin antagonistisch wirken, selbst die Größenordnung der wirksamen Dosen unterscheidet sich kaum (OrLszNER unpubliziert).

In unseren Versuchen konnte der für die Tremorinhypothermie bereits bekannte Befund der Unwirksamkeit der Phenothiazinderivate, die gegen den Tremor wirksam sind, auch für die Temperatursenkung nach Arecolin nachgewiesen werden. Das ist unseres Erachtens ein Hinweis darauf, daß dem Tremor und der Hypothermie pharmakologisch-differenzierbare Mechanismen zugrunde liegen. Der Tremor wird durch die Phenothiazine offenbar über einen Angriffspunkt beeinflußt, der tremorspezifisch, aber verschieden von dem der zentralen Cholinolytika ist.

Der mit hohen Dosen Methylatropin bei Arecolin erzielbare antihypotherme Effekt schließt eine vorwiegend zentrale Genese der Thermoregulationsstörungen nicht aus, da bei diesen Dosen eine Permeation durch die Blut-Hirn-Schranke angenommen werden kann (Paul-David, Riehl \& Unna 1960). Ein rein peripherer Mechanismus der Störung der Wärmeregulation nach Arecolin, etwa durch primäre Kreislaufeffekte ( $v$. Euler \& DoMer 1945), die infolge der peripher muskarinartigen Wirkungen des Arecolins diskutiert werden könnten, ist deshalb ebenso wie für Tremorin beziehungsweise dessen aktivem Metaboliten Oxotremorin unwahrscheinlich. Eine Beteiligung einer solchen Komponente kann jedoch nicht völlig abgelehnt werden, zumal auch peripher wirkende Cholinergica wie Carbaminoylcholin zu einer Kerntemperatursenkung an der Ratte führen (FESZT 1964).

Die Ergebnisse der vorliegenden Untersuchungen können dahingehend gedeutet werden, daß die durch Arecolin auslösbare Hypothermie qualitativ nicht von der Körpertemperatursenkung nach Tremorin unterscheidbar ist. Beide sind durch einen vorm wiegend zentralen Mechanismus bedingt und können durch zentrale Cholinolytika hemmend beeinflußt werden. Es liegen Hinweise darauf vor, daß die Hypothermie einerseits und die zentral bedingten motorischen Effekte andererseits sowohl bei Tremorin als auch bei Arecolin auf einem cholinergen Mechanismus beruhen. Aus der Unwirksamkeit der tremorantagonistischen Phenothiazinderivate ergibt sich eine Differenzierungsmöglichkeit der Angriffspunkte von Antiparkinsonmitteln.

\section{ZUSAMMENFASSUNG}

1. Arecolin ruft bei Mäusen, Ratten und Meerschweinchen eine der Tremorinhypothermie vergleichbare Senkung der Körpertemperatur hervor.

2. Die hypothermiewirksamen Dosen des Arecolins sind bei der Maus in Abhängigkeit 
von der Umgebungstemperatur bei intraperitonealer Gabe 5- bis $10 \mathrm{mal}$ höher als die isoeffektiven Tremorindosen.

3. Der Hypothermieeffekt beider Stoffe kann dosisabhängig durch Atropin, Scopolamin, Benactycin und Trihexyphenidyl gehemmt werden.

4. Die arecolin- und tremorinbedingte Hypothermie wird durch auf die motorischen Effekte beider Stoffe hemmend wirkende Phenothiazine Diaethazin, Promethazin und Aethopropazin nicht vermindert.

5. Die nur in hohen Dosen in das Zentralnervensystem eindringenden quaternären Verbindungen Methylatropin und Butylscopolamin beeinflussen in Dosierungen, die mit den wirksamen Mengen von Atropin und Scopolamin vergleichbar sind, die Senkung der Körpertemperatur hingegen nicht.

6. Arecolin- und Tremorinhypothermie werden vorwiegend zentral über einen cholinergen Mechanismus ausgelöst.

Für die Mitarbeit bei der Durchführung der Versuche sei an dieser Stelle den medizinischtechnischen Assistentinnen Fräulein E. SCHLENKER und Fräulein G. BAunack gedankt.

\section{ZITIERTE LITERATUR}

AdLER, K., 1964. Die Wirkung von Sympathomimetica und -lytica auf den durch Arecolin erzeugten Tremor der Maus. Dresden, Med. Diss.

Euler, U. S. von \& Domerj, B., 1945. Nicotine-like actions of arecoline. Acta pharmac. tox. 1, 263-269.

Everett, G. M., Blockus, L. E. \& Shepperd, J. M., 1956. Tremor induced by tremorine and its antagonism by anti-Parkinson drugs. Science, N.Y.124, 79 .

- 1964. Pharmacological studies on tremorine. Int. pharmac. Meet. (Prag 1963) 2, 69-74.

Farquharson, M. E. \& Johnston, R. G., 1959. Antagonism of the effects of tremorine by tropine derivatives. Br. J. Pharmac. Chemother. 14, 559-566.

Ferrari, W. \& Gessa, G. L., 1964. Failure of anti-Parkinson drugs to antagonize hypothermia in tremorine-treated rats. Int. pharmac. Meet. (Prag 1963) 2, 105-107.

FESZT, G., 1965. Réaction hypothermique conditionelle liée à l'effet de la carbaminoylcholine. Experientia 21,98.

Healy, S. T. \& Jenney, E. H., 1959. Drug antagonists to arecoline inhibition of the conditioned response. Fedn Proc. Fedn Am. Socs exp, Biol. 18, 400.

Herz, A., 1962. Wirkungen des Arecolins auf das Zentralnervensystem. Arch. exp. Path. Pharmak. 242, 414-429.

Keranen, G. M., Zaratzian, V. L. \& Coleman, R., 1961. Studies on 1,4-Dipyrrolidino-2butyne (tremorine) in mice. Toxic. appl. Pharmac. 3, 481-492.

Litchfiel. J. J. T. \& WiLcoxon, F., 1949. A simplified method of evaluating dose-effect experiments. J. Pharmac. exp. Ther. 96, 99-113.

Matckel, R. P., Stern, D. M. \& Brodie, B. B., 1964. The role of autonomic nervous function in mammalian thermoregulation. Int. Pharmac. Meet. (Prag 1963) 2, 225-237.

Paul-David, J., Rieht, J. L. \& UnNA, K. R., 1960. Quantification of effects of depressant drugs on EEG activation response. $J$. Pharmac. exp. Ther, 129, 69-74.

Pepeu, G., 1963. Effect of tremorine and some anti-Parkinson's disease drugs on acetylcholine in the rat's brain. Nature, Lond. 200, 895.

Votova, Z., 1962. The effect of some cholinolytic agents and tranquilizers on the duration of nicotine and arecoline-induced spasms in rabbits. Pharmac. Toxic. 25, 291 


\section{Diskussion im Anschluß an den Vortrag STAIB}

BRÜCK: Beruht die Hypothermie nach Tremorin und Arecolin auf einer Reduktion der muskulären Wärmebildung oder auf einer Erhöhung der Wärmeabgabe durch Beeinflussung der peripheren Vasomotorik?

StArB: Die Effekte von Tremorin und Arecolin auf die Motorik können nicht ohne weiteres mit der Beeinflussung der Thermoregulation in Verbindung gebracht werden, da Tremor etc. auch bei solchen Umgebungstemperaturen in praktisch gleicher Intensität auftreten, bei denen die Körpertemperatur unbeeinflußt bleibt $\left(+30^{\circ} \mathrm{C}\right.$ Umgebungstemperatur). Der Tremor ist also nicht als Gegenregulation eines auskühlenden Organismus deutbar. Durch Tremorin und Arecolin wird nach unserer Meinung über eine zentrale Auslösung die Wärmeabgabe erhöht. Es dürfte also vorwiegend die physikalische Wärmeregulation gestört sein. 